\title{
Instrumentos para avaliação do uso de grades laterais do leito: segurança ou contenção?
}

\begin{abstract}
RESUMO | Objetivo: Identificar na literatura os instrumentos para avaliação do uso de grades em pacientes hospitalizados e intervenções alternativas para reduzir as quedas do leito sem o uso de grades. Método: Revisão integrativa conforme diretrizes PRISMA, nas bases de dados: LILACS, Health Literature, MEDLINE/PUBMED, SCOPUS e SCIENCE DIRECT sem recorte temporal nos idiomas português, inglês e espanhol. Critérios de inclusão: população (adultos e idosos), intervenção (prevenção de quedas), desfecho (Instrumento de avaliação). Resultados: Foram selecionados quatro artigos que usavam um mesmo instrumento "Evaluation of Siderail Use" com abordagem detalhada na avaliação dos pacientes quanto ao uso de grades, discorre intervenções alternativas para o uso de grades que devem ser adaptadas às necessidades individuais. Conclusão: Estudos recomendam avaliação sistemática no processo de decisão sobre o uso das grades, que direcionem a prática clínica do enfermeiro para decidir por levantar ou não as grades, minimizando o uso desnecessário das grades como contenção mecânica.
\end{abstract}

Palavras-chaves: restrição física; assistência hospitalar; segurança do paciente.

\begin{abstract}
Objective: Identify in the literature the instruments to evaluate the use of siderail in hospitalized patients and alternative interventions to reduce falls without use bedrail. Method: It was conducted an Integrative review of the literature according to the PRISMA guideline, carried out in the databases: LILACS, CINAHL, MEDLINE / PUBMED, SCOPUS and, SCIENCE DIRECT without temporal cut and in the idioms Portuguese, English and Spanish. Inclusion criteria: population (adults and the elderly), intervention (evaluation of the use of siderails), outcome (siderails use assessment tool); Exclusion criteria: articles of children and that did not deal with evaluation of the use of siderails. Results: It was selected four articles that used as intervention evaluations instruments for use of siderail. Conclusion: It was possible to verify a gap in the studies that approach instruments of evaluation of the use of siderail in hospitals. In addition, it is necessary to provide instruments to nurses to educate and train teams to decrease or eliminate the use of siderail as containment.
\end{abstract}

Keywords: restraint physical; hospital care; patient safety.

RESUMEN I Objetivo: identificar en la literatura las herramientas para evaluar el uso de rejillas en pacientes hospitalizados y de intervenciones alternativas para reducir las caídas de la cama sin el uso de rejillas. Método: revisión Integrativa como directrices, bases de datos de prisma: LILACS, literatura de la salud, MEDLINE/PUBMED, SCOPUS y SCIENCE DIRECT sin temporal en los idiomas portugués, Inglés y español. Criterios de inclusión: población (adultos y mayores), intervención (prevenir caídas), cierre (HABILITACION). Resultados: se seleccionaron cuatro artículos utilizando un mismo instrumento "Evaluación de Siderail uso" con enfoque detallado en la evaluación de pacientes con respecto al uso de las redes, direcciones alternativas para el uso de barras de las intervenciones que se deben adaptar necesidades individuales. CONCLUSIÓN: Estudios recomiendan evaluación sistemática en la toma de decisiones sobre el uso de barras, que dirigen la práctica clínica de enfermería para decidir si o no subir las barras, reduciendo al mínimo el uso innecesario de rejillas como retención mecánica.

Descriptores: : restricción física; Asistencia de hospital; Seguridad del paciente.

\section{Teresa Cristina Brasil Ferreira}

Enfermeira coordenadora da CME (Central de Material e Esterilização) do Hospital Municipal Miguel Couto/ Secretaria Municipal de Saúde do Rio de Janeiro. Coordenação do Núcleo de Segurança do Paciente. Membro da câmara técnica de Enfermagem do Município do Rio de Janeiro. Atuou como preceptora no projeto PETSaúde (PUC-Rio). Mestranda do Mestrado Profissional em Enfermagem Assistencial/ UFF-RJ.

\section{Rosimere Ferreira Santana}

Pós-doutorada. Bolsista de Produtividade do CNPq PQ2. Professora Associada da Universidade Federal Fluminense, Coordenadora do Programa de Mestrado Profissional em Enfermagem Assistencial. Coordenadora do Departamento Cientifico de Enfermagem Gerontológica (DCEG) da ABEn- Nacional . Coordenadora do Acordo de Cooperação entre a UFF e a Azienda per Lassistenza Sanitaria Triestina - Itália pela Cultura da Não Contenção.

\section{Thalita Gomes do Carmo}

Doutora em Ciências do Cuidado da Saúde (PACCS/UFF). Professora da Escola de Enfermagem Aurora de Afonso Costa da Universidade Federal Fluminense Departamento Médico Cirúrgica (MEM). Professora do MBA Gestão em Saúde e Controle de Infecção.

\section{Marcia Veríssimo de Souza}

Mestre em Enfermagem Assistencial. PósGraduação Lato Sensu em Enfermagem Dermatológica. Coordenadora de Enfermagem no Serviço de Hemoterapia (Hemocentro) do Hospital Universitário Antônio Pedro, enfermeira da Comissão Terapêutica de Feridas do Hospital Federal dos Servidores do Estado do Rio de Janeiro.

\section{Lívia Maria da Silva Souza \\ Mestre em Enfermagem Assistencial. Residência em clínica médica. Docente do curso de Enfermagem da Universidade Estácio de Sá. Professora substituta na Universidade Federal do Rio de Janeiro.}

Recebido em: 13/02/2019

Aprovado em: 13/05/2019 


\section{INTRODUÇÃO}

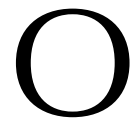
eventos adversos (EA) são incidentes que resultam em dano à saúde e são também definidos como injúrias não intencionais decorrentes do cuidado prestado aos pacientes ${ }^{1}$, não relacionadas à evolução natural da doença de base e que, obrigatoriamente, acarretam lesões mensuráveis nos pacientes afetados, óbito, ou prolongamento do tempo de internação².

A ocorrência de eventos adversos passou a ser discutida como um dos aspectos que impactam negativamente a segurança do paciente, principalmente a partir de 1999, com a divulgação do relatório "To Err is Human" pelo Institute of Medicine ${ }^{3}$. Nesse relatório apontou-se os prejuízos financeiros, aumento da permanência do paciente no hospital, necessidade de intervenção diagnóstica e terapêutica, e até mesmo a morte.

Estudos demonstram que as lesões dos pacientes associadas aos cuidados de saúde aumentam substancialmente os custos, estimando-se entre 13 e $16 \%$ dos custos hospitalares ${ }^{4}$. Logo, a Segurança do paciente foi uma política e prática instituída para redução dos riscos de danos desnecessários associados à assistência à saúde até um mínimo aceitável ${ }^{5}$.

Segundo estudos conduzidos em hospitais americanos há uma estimativa de que aproximadamente uma em cada dez admissões hospitalares resulta em pelo menos um evento adverso, ainda mais se considerar que metade desses incidentes poderiam ter sido evitados6. No Brasil, estudo realizado em três hospitais de ensino evidenciou a incidência de eventos adversos de $7,6 \%$, dos quais $66,7 \%$ foram considerados evitáveis ${ }^{7}$.

Desde 2004 o tema Segurança do Paciente vem sendo desenvolvido sistematicamente pela ANVISA (Agência Nacional de Vigilância Sanitária), e tem sido reforçado com a publicação das diretrizes estabelecidas pelo Programa Nacional de Segurança do Paciente (PNSP). Para institucionalizar as práticas seguras a ANVISA instituiu a RDC no 36, de 25 de julho de 2013, que determinou aos estabelecimentos de saúde que construíssem os planos locais de segurança do paciente através da formação das Comissões que são os Núcleos de Segurança do Paciente'. Dentre os protocolos citados destacam-se as quedas que resultam em danos e são um problema prevalente de segurança do paciente.

Os idosos e pacientes frágeis com risco de queda não são os únicos que possuem vulnerabilidade de queda nos estabelecimentos de saúde. Qualquer paciente e de qualquer idade ou habilidade física pode ter risco de queda devido fatores como condição médica, medicações, cirurgias, procedimentos ou diagnóstico ${ }^{8}$.

Medidas de contenção têm sido utilizadas para prevenir quedas, controlar pacientes com distúrbio de comportamento ou motor e para permitir a continuidade do tratamento principalmente em medicina geriátrica e em idosos ${ }^{9-10}$. No cuidado domiciliar a contenção mecânica dos residentes é realizada principalmente pelo uso de grades ${ }^{9-10}$.

O uso de medidas de contenção mecânica contra a vontade do paciente pode ser uma intrusão aos direitos humanos básicos ${ }^{11}$. Além disso, o uso indevido de contenções mecânicas pode causar ferimentos de gravidade variável, o que às vezes pode ser fatal ${ }^{11}$. Grades laterais do leito também são consideradas uma forma de contenção quando restringem a liberdade de movimento e impedem que a pessoa saia da cama quando desejar, independentemente da capacidade de fazer isto com segurança ${ }^{12}$. As grades têm sido correlacionadas com eventos adversos que incluem agitação, incontinência urinária e fecal, danos e mortes por aprisionamento $^{13-18}$. Um estudo conduzido em um hospital da Nova Zelândia encontrou uma diminuição no número de quedas e danos após a implementação de uma política para reduzir o uso de grades $^{19}$.

$\mathrm{O}$ Food and Drug Administration (FDA)20, órgão federal dos Estados Unidos que regula o uso de alimentos, medicamentos e dispositivos médicos entre outros, estipulam que as grades não deveriam ser usadas como contenção mecânica. Entretanto, evidências na literatura sugerem que este fato tem sido ignorado com frequência, pois as grades são comumente usadas como contenção ${ }^{21-22}$ e tem sido usadas indiscriminadamente na prática do Reino Unido $^{13,23-24}$, o que por sua vez pode comprometer a segurança, a dignidade e a autonomia do paciente ${ }^{25}$.

Os danos são mais frequentes quando, por exemplo, os pacientes tentam escalar as grades para sair da cama ${ }^{17-18}$. Estudos analisaram as diferenças na incidência de queda com o aumento no uso de grades e a diminuição do uso. Dentre esses, no estudo de Grasso et $\mathrm{al}^{26}$, os autores observaram que a incidência de quedas do leito com grades elevadas foi igual ou maior do que quando as grades não estavam elevadas. Os pacientes em um estado "não racional" no momento da queda foram significativamente mais propensos a ter caído com as grades elevadas ${ }^{26}$.

Em outro estudo reduzir o uso de grades não diminuiu o número de quedas do leito e resultou em significativamente menos lesões graves ${ }^{27}$. O aumento do uso de grades não resultou em diminuição de quedas, quedas recorrentes ou ferimentos graves $^{27}$. Por isso, é indispensável realizar uma avaliação do uso de grades ${ }^{28}$ e criar de medidas que contemplem a avaliação de risco aos quais os pacientes estão submetidos.

Tendo em vista à atualidade do tema e a relevância de se introduzir na cultura de segurança do paciente no Brasil o uso de grades do leito como contenção mecânica, elaborou-se como per- 
gunta: Quais são os instrumentos para avaliação do uso de grades do leito em pacientes hospitalizados que auxiliam na prevenção de quedas sem uso da contenção mecânica? E como objetivo: Identificar na literatura os instrumentos para avaliação do uso de grades em pacientes hospitalizados e intervenções alternativas para reduzir as quedas do leito sem o uso de grades.

\section{MÉTODO}

Trata-se de revisão integrativa de literatura, esse tipo de estudo inclui a análise de pesquisas relevantes que dão suporte para a tomada de decisão e a melhoria da prática clínica, possibilitando a síntese do estado do conhecimento de um determinado assunto. Além disso, permite apontar lacunas do conhecimento que precisam ser preenchidas, bem como a sintetiza dos estudos publicados conclusões gerais a respeito de uma particular área de estudo ${ }^{7}$.

\section{Figura 1. Fluxograma Prisma de coleta e análise dos dados}

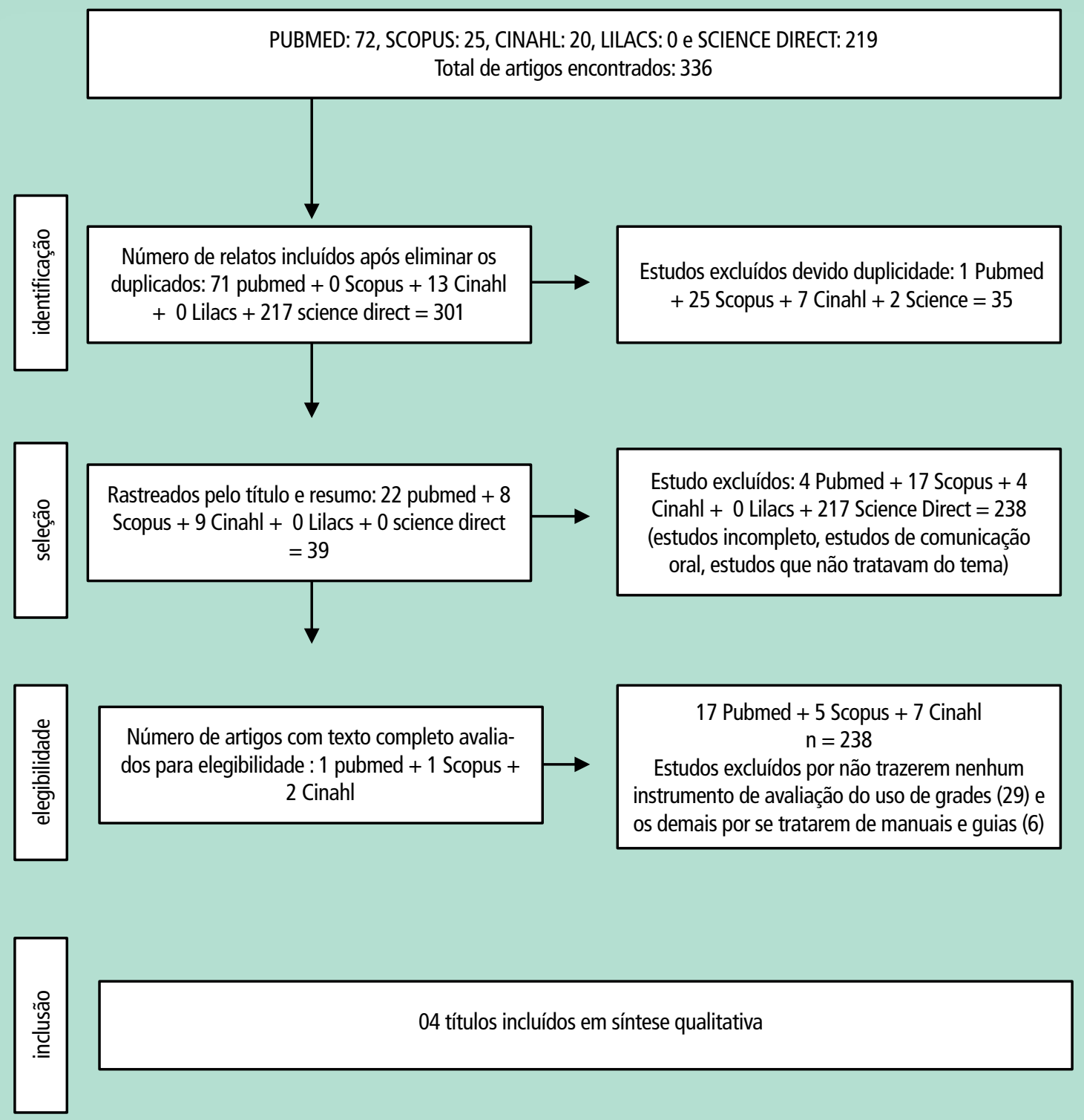


Para atender aos requisitos do método seis etapas foram seguidas: (1) identificação do tema; (2) seleção da questão de pesquisa; (3) definição dos critérios para inclusão e exclusão dos estudos e das informações a serem extraídas; (4) categorização e avaliação dos estudos incluídos na revisão; (5) interpretação dos resultados e (6) apresentação da revisão/ síntese do conhecimento ${ }^{7}$.

Utilizou-se da estratégia PICO (acrônimo P: para população - pacientes hospitalizados; I para intervenção medidas de prevenção de quedas sem contenção mecânica; Co para contexto: uso de grades no leito) para a elaboração da pergunta: Quais são os instrumentos para avaliação do uso de grades do leito em pacientes hospitalizados que auxiliam na prevenção de quedas sem uso da contenção mecânica ${ }^{36-38}$.

Com a questão de pesquisa formulada definiu-se os critérios de inclusão dos artigos: a) artigos das bases de dados: Literatura Latino - Americana em Ciências da Saúde (LILACS), Cumulative Index to Nursing and Allied Health Literature (CINAHL), Literatura Internacional em Ciências da Saúde (MEDLINE/PUBMED), SCOPUS, SCIENCE DIRECT sem recorte temporal, b) utilização das palavras-chave isolada e aleatoriamente em cada base de dados: bedrail; siderails; side rail; siderail e siderail.

A busca foi realizada sem recorte temporal utilizando três idiomas: português, inglês e espanhol, porém somente artigos em inglês foram encontrados.

Os artigos identificados pela estratégia de busca inicial foram avaliados independentemente por dois autores, conforme os seguintes critérios de inclusão: (1) população (adultos e idosos), (2) intervenção (avaliação do uso de grades), (3) desfecho (Instrumento de avaliação do uso de grades).

O processo de busca e a seleção de material estão representados no fluxograma Prisma da Figura 1. Dos 336 artigos identificados, foram excluídos 10 artigos duplicados. Após essa etapa 301 artigos foram identificados, 238 estudos foram excluídos (estudos incompletos, estudos de comunicação oral, estudos que não tratavam do tema) e 39 foram selecionados para leitura completa. Após os critérios de elegibilidade 29 estudos artigos foram excluídos por se tratarem de Manuais e/ou Guias, que apesar de relevantes não eram considerados artigos científicos, portanto, a amostra final foi composta por quatro artigos.

Para a coleta de dados foi utilizado um instrumento que abordava os itens: autor, ano, base de dados/ tipo de material/ revista/local, desfecho/instrumento. Na avaliação dos artigos foi realizada a leitura completa de cada um, para análise global e individual dos instrumentos de avaliação do uso de grades do leito disponíveis na literatura.

Tabela 1. Contingente de artigos idenficados na Revisão Integrativa, Rio de Janeiro, 2019.

\section{AUTOR}

ANO

Laura M. Wagner, Elizabeth Capezuti, Barbara Brush, Marie Boltz, Susan Renz, Karen A. Talerico.

\section{BASE DE DADOS/ TIPO DE MATERIAL/ REVISTA/ LOCAL}

Cinahl/ artigo cientifico/

Research in nursing \& health

Canadá
TíTULO

Description of an advanced practice nursing consultative model to reduce restrictive siderail use in nursing homes.
Elizabeth Capezuti, Laura M. Wagner, Barbara L. Brush, , Marie Boltz, Susan Renz, and Karen A. Talerico,

Elizabeth Capezuti, Karen

A. Talerico, Neville Strumpf, Lois Evans,

1999

2007

Pubmed/artigo/cientifico/

The American Geriatrics Society/ EUA

Cinahl/artigo científico/
Geriatric Nursing
EUA

Individualized Assessment ral Siderail Use
Consequences of an Intervention to Reduce Restrictive Side Rail Use in Nursing Homes
DESFECHO/ INSTRUMENTO
Elizabeth Capezuti, Karen A Talerico, Ina Cochran, Honore Becker, Neville 1999 Cinahl/artigo científico/ Journal of gerontological nurse/ EUA Strumpf, Lois Evans
"Evaluation of siderail Usage"

"Evaluation of siderail Usage"
Individualized Interventions to prevent bed-related falls and reduce siderail use

\section{"Individualized Assessment for Evaluation of siderail Usage"}

Aponta cinco categorias de problemas que frequentemente resultam em uso de grades: "distúrbio de memória, mobilidade prejudicada, risco de lesões, nocturia/incontinência e distúrbio do sono".

Fonte: a própria autora 
RESULTADOS

Foram incluídos no estudo quatro artigos que abordavam instrumentos de avaliação individualizada do uso de grades laterais em residentes de Instituições de Longa Permanência de Idosos, esses estão detalhados na Tabela 1.
De acordo com os pesquisadores, nenhuma intervenção isoladamente representa uma solução para o uso de grades, existe uma variedade de inter-

Quadro 1 - Contingente de Intervenções sumarizadas na revisão integrativa para prevenir quedas, Rio de Janerio, 2019.

Problema

Distúrbio de memória

Mobilidade Prejudicada

Potencial para lesões

Noctúria/ Incontinência

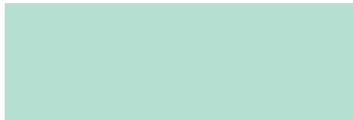

Distúrbio do sono
Remover barreiras como grades laterais de comprimento total e estimular uso de dispositivos auxiliares, como um andador ou mesa de cabeceira.

Amplitude de movimento (ROM): utilizar fisioterapia, terapia ocupacional com exerć́cios de força e ortostáticos. Cuidados de reabilitação para contraturas e/ou diminuição do ROM;

Aumentar a consciência dos profissionais para:

a) Utilizar campainhas de chamada sensíveis à luz;

b) Utilizar interfones ou monitores tipo babá eletrônica para quartos/leitos ou banheiros;

c) Identificar as portas dos quartos/ enfermarias pacientes de alto risco de queda com sinalizações coloridas para alertar a equipe que eles precisam de cuidados especiais.

d) Posicionar alarmes para detectar movimentos dos pacientes sob o colchão.

Atentar para pacientes de baixa estatura: ajustar ou personalizar a altura dos leitos para pacientes de baixa estatura ( mais baixos) e consultar o terapeuta ocupacional para ajustar o assento do banheiro para este tipo de paciente de baixa estatura.

Facilitar deambulação ou transferência:

a)Utilize o trapézio ou $1 / 2$ grade ou $1 / 4$ de grade no lado que o paciente tem mais força.

b)Adicione um facilitador de transferência ao quadro da cama para aumentar a firmeza do colchão.

c)Os residentes devem vestir roupas fáceis de serem tiradas ou pijamas, à noite;

d) Use chinelos, tapetes e piso antiderrapantes.

e) Crie um caminho iluminado e livre de móveis no trajeto do residente ao banheiro. Coloque mais interruptores para que ele possa acender as luzes.

Dor

Procure avaliação médica e de Enfermagem, revisão farmacológica e programação de analgésicos.

Potencial para lesão

a) Utilize demarcadores de limites na cama: coxins nas laterais, colchões côncavos, cobertores enrolados ou macarrões de natação debaixo dos lençóis;
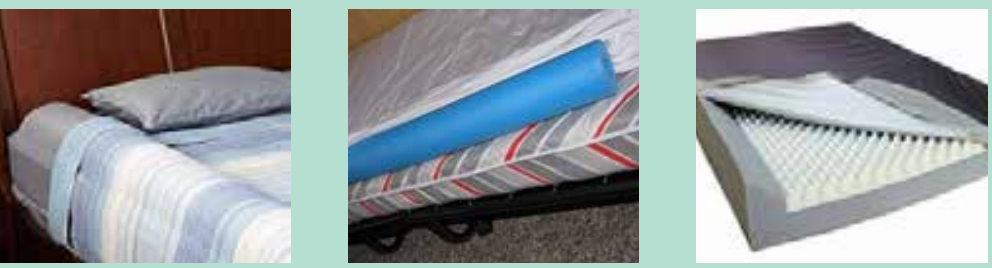

Prevenção Primária: procure avaliar a incontinência urinária e realizar rounds para eliminação individualizada. Use comadres e patinhos ou fraldas super absorventes para evitar que o residente fique molhado.

Diminua o risco de queda: Posicione uma mesa de cabeceira no lado em que o residente tem mais força. Indique sapatos antiderrapantes com solado de borracha. Identifique os banheiros com fotos ao invés de palavras.

Insônia: Procure reavaliar a prescrição médica e farmacológica. Utilize o "ruído branco" para promover relaxamento. Ruído branco é um sinal sonoro que contém todas as frequências na mesma potência como o som da TV quando não está sincronizada. (Esse barulho faz com que o limiar auditivo atinja seu nível máximo, o que significa que, na presença desse tipo de som, os estímulos auditivos mais intensos têm menos capacidade de ativar o córtex cerebral durante 0 sono).

b) Procurar manter o ciclo regular do sono e evitar cochilos diurnos excessivos.

Conforto: Use almofadas; utilize grades somente com o consentimento do residente/família e programe analgésicos. Integridade da pele: Usar colchões de ar ou outra alternativa para alívio da pressão como coxins e almofadas. 
venções adaptadas às necessidades individuais. O processo de seleção dessas intervenções individualizadas para reduzir as quedas do leito foram sintetizadas no Quadro 1.

\section{DISCUSSÃO}

Como principal achado do estudo detectou-se que os artigos que emergiram da revisão são antigos, de 1999 e 2007, portanto, a necessidade de pesquisas sobre a temática, visto a crescente implantação das políticas de segurança do paciente que recomendam medidas de prevenção de quedas enfaticamente ${ }^{29}$.

Os quatros artigos abordam sobre instrumentos de avaliação do uso de grades, porém os artigos traziam apenas instrumentos de avaliação de grades utilizados em Instituições de Longa Permanência de Idosos (ILPI), não encontramos nenhum instrumento utilizado na área hospitalar, apesar das autoras recomendarem estudos testando as escaldas em outros ambientes de cuidado.

Capezuti $^{27}$ (1998) em seu estudo avaliou a efetividade de um modelo de Prática Avançada de Enfermagem, na redução do uso de grade como contenção bem como das quedas em Casas de Repouso ou Instituições de Longa Permanência (ILPI). Para isso foi utilizado uma escala de avaliação individualizada "Evaluation of Siderail Use". O instrumento desenvolvido promove uma abordagem detalhada na avaliação dos pacientes e sua adequação ao uso de grades. O instrumento requer que a enfermeira preencha um formulário de entrevista com pacientes e considera o estado físico, psicológico e os fatores ambientais.

Em outro estudo a mesma autora Capezutti et l $^{30}$ avaliou a relação entre a prática avançada de Enfermagem com a redução do uso de grades e com o custo das alternativas para reduzir o uso de grades em 273 residentes em 4 casas de repouso na Filadélfia. Foram recomendadas 1275 alternativas de intervenção, com custo médio de 135 doláres, com o objetivo de redução das quedas. Evidenciou-se, assim que o modelo de consulta baseado na Prática Avançada de Enfermagem pode ser implementado através de uma avaliação individualizada sem trazer custos substanciais para a instituição.

A justificativa para considerar as grades como uma forma de manter a segurança do paciente deve ser ponderada entre os benefícios e os riscos inerentes ao uso das grades laterais do leito. É razoável sugerir que grades devam ser utilizadas somente quando apresentem riscos minimizados em relação a intervenções alternativas a contenção. Portanto, essa avaliação abrangente pode superar o uso indiscriminado de grades e auxiliar a equipe de saúde, pacientes e familiares a decidirem por intervenções menos restritivas ${ }^{22}$.

Donius e Rader criaram uma árvore de decisão sobre grades laterais abrangendo três níveis de avaliação de risco de quedas ${ }^{11}$. Porém, não deixam claro sobre os pacientes que tem capacidade de se levantar da cama, que necessitam de ajuda e não traz perguntas relativas ao passado de pacientes que já tentaram escalar as grades e ou que tem o risco de fazer isso novamente. Entretanto, caso uma escala com esses itens fosse utilizada na prática clínica dos enfermeiros, esses fatores passariam a ganhar prioridade.

Os princípios da avaliação de grades da cama planejados por Bride Everitt e Bridel- Nixon²4, 1997 são baseados numa revisão de literatura sobre risco de queda. O instrumento foi avaliado na avaliação de conteúdo por peritos como compreensível, específico e breve. Os autores também produziram orientações sobre o uso das grades laterais como complemento a essa avaliação. $O$ instrumento foi aprimorado pela adição de uma pontuação para os fatores de risco apresentados, como isso a abordagem utilizada é baseada em evidências e auxilia o trabalho dos enfermeiros em qualquer especialidade.

No estudo de Capezuti ${ }^{30}$ os autores relatam cinco categorias de problemas que resultam em uso de grades laterais: distúrbio de memória, mobilidade reduzida, risco de lesões, noctúria/incontinência e distúrbios do sono. Como as casas de idosos ou Instituições de longa Permanência (ILPI) precisam trabalhar para atender ao que determina o HCFA (Administração Financeira de Cuidados Médicos) sobre as grades laterais, os administradores e profissionais precisam implementar intervenções que ofereçam segurança e que individualizem os cuidados. As diretrizes do HCFA orientam que as Instituições de Longa Permanência desenvolvam planos de cuidados que atendam às necessidades individuais de segurança dos residentes. Ou seja, as grades devem ser indicadas individualmente, e não grades para todos os pacientes idosos.

Segundo Foltz-Gray ${ }^{31}$ o processo de avaliação e a justificativa para o uso de cada intervenção de prevenção de quedas precisam ser documentados. A redução das grades laterais requer além de suporte administrativo, políticas que reflitam sobre a não contenção ou ao menos para a mínima contenção e recursos financeiros para a compra de novos equipamentos, recursos humanos qualificados e uma política de inclusa da família nos cuidados em saúde.

Grande parte das intervenções sugeridas tem um baixo custo, com exceção para aquelas que previnem alto risco de lesões como a cama baixa que pode ser uma aquisição mais onerosa. Pesquisas demonstram redução de custos com a prevenção de lesões relacionadas as grades, além de prevenir as quedas no leito. É necessário encorajar as instituições de saúde à adotarem uma redução significativa de grades.

Capezutti et $\mathrm{al}^{27}$ detalha a importância de avaliações individualizadas 
para implementar as intervenções de redução do uso de grades laterais do leito. Os autores acreditam que o uso de grades como contenção pode ser reduzido por um processo de avaliação individualizada da necessidade das grades laterais, além de orientar os enfermeiros no manejo do paciente de alto risco. Esse instrumento traz que a avaliação individualizada consiste na coleta de dados em quatro áreas: história do residente (razões para uso de grades, registros do cuidado e incidentes reportados); exame físico; características do ambiente (quarto e banheiro); problemas específicos do residente. A partir daí deve ser desenvolvido o plano de cuidados para minimizar ou eliminar o uso de grades.

Em um artigo de intervenção para diminuir o uso de grades como contenção em 4 casas de repouso e com uma amostra de 251 residentes, fez a associação entre grades laterais e as quedas da cama. A hipótese do estudo foi que a redução no uso das grades como contenção pode ter sucesso sem comprometer a segurança do residente, ou seja, não aumentou o numero de incidentes por queda, ao contrario diminui, pois, a equipe de saúde sabiam os riscos individuais, e implementaram estratégias de prevenção de riscos. A intervenção empregou estratégias destinadas a ajudar os profissionais da Instituição de Longa Permanência de Idosos a desenvolver habilidades no processo de avaliação e manejo da redução da contenção por grades laterais ${ }^{30}$. Uma enfermeira gerontóloga com mestrado de EPA realizou a avaliação de cada paciente após o consentimento deles. Para isso utilizou o instrumento "Evaluation of siderail Usage"27 para identificar os fatores de risco que influenciavam no uso de grades (queda da cama, mobilidade na cama, habilidade de transferência, problemas com sono e nível de continência. Após as avaliações e recomendações (intervenções) para diminuir o uso de grades foi realizado um plano de cuidados $^{27}, 32$ revisado com enfermeiros e outros profissionais da unidade. O resultado apontou redução do uso de grades, assim como da contenção mecânica pode se ter cumprido com sucesso as medidas de intervenção, sem comprometer a segurança dos participantes do estudo.

O uso de grades laterais foi identificado como restritivo e não restritivo. As grades como contenção/restritivas foram designadas como uma grade de comprimento inteiro levantada ou quatro grades de meio comprimento levantadas e não restritivo para nenhuma grade levantada, uma grade de comprimento completo levantada de um lado ou uma a três grades de meio comprimento levantadas. Os achados apontaram que a redução do uso de grades foi associada com a diminuição do número de quedas da cama, assim como a diminuição da contenção mecânica. O uso persistente de grades laterais reflete um consenso gradual e equivocado, em vez de uma prática orientada empiricamente ${ }^{1}$.

Em suma os estudos descrevem que uma avaliação individualizada e sistemática no processo de decisão sobre o uso das grades contribui para uma prática avançada de enfermagem ${ }^{12,24,27-28,34}$. Além de fazer com que os enfermeiros reflitam de forma abrangente sobre os desejos dos pacientes e familiares em relação ao uso das grades laterais, bem como sobre o estado físico, cognitivo, sensorial e de mobilidade, os medicamentos utilizados, o histórico do paciente e os fatores ambientais $^{22}$. A evidência desses estudo $^{19,27,35}$ juntamente com as consequências negativas associadas a contenção mecânica questionam seu papel nas instituições de saúde como uma intervenção de enfermagem.

\section{CONCLUSÃO}

A partir dessa revisão identificou-se a inexistência de estudos nacionais que abordam instrumentos de avaliação do uso de grades e, que possam de direcionar a prática clínica do enfermeiro para decidir por levantar ou não as grades laterais do leito. O estudo contribui para a mudança de paradigma de que as grades são um dispositivo para a proteção do paciente, além de instrumentalizar os enfermeiros para educação e formação das equipes, minimizando/eliminando o uso desnecessário de grades como contenção mecânica. Instrumentos de avaliação do uso de grades são indispensáveis para a elaboração de um plano de cuidados dos enfermeiros, favorecendo a acurácia diagnóstica e a intervenções de enfermagem individualizadas, garantindo aos pacientes dignidade, autonomia e independência.

\section{Referências}

1. ANVISA. RDC n³6 de julho de 2013. Institui ações para a Segurança do Paciente em serviços de saúde e dá outras providências. Agência Nacional de Vigilância Sanitária [Internet], Brasília (DF). Disponível em: http://bvsms.saude.gov.br/bvs/saudelegis/anvisa/2013/rdc0036_25_07_2013.html 2. Hiatt $H H$, Barnes BA, Brennan TA, Laird NM, Lawthers AG, Leape $L L$, et al. A study of medical injury and medical malpractice. N Engl J Med [Internet]. 1989 Ago [citado 2018 Nov 23]; 321(7):480-4. Disponível em: https:// www.nejm.org/doi/pdf/10.1056/NEJM198908173210725

3. Institute Of Medicine. To err is human: building a safer health system. Washington (DC): National Press Academy; 1999. 


\section{Referências}

4. Jackson T. One dollar in seven: scoping the economics of patient safety: a literature review prepared for the Canadian Patient Safety Institute. Alberta: Canadian Patient Safety; 2009.

5. Organização Mundial de Saúde (OMS). The conceptual framework for the international classification for patient safety. Final Technical Report and Technical Annexes, 2009. Disponível em: http://www.who.int/patientsafety/taxonomy/en/

6. Watcher RM. Patient safety at ten: unmistakable progress, troubling gaps. Health Affairs [Internet]. 2010 [citado 2018 Nov 23]; 29(1):16573. Disponível em: https://www.healthaffairs.org/doi/full/10.1377/hlthaff.2009.0785

7. Mendes KDS, Silveira RCCP, Galvão $C M$. Revisão integrativa: método de pesquisa para a incorporação de evidências na saúde e na enfermagem. Texto e Contexto Enfermagem [Internet]. 2008 [citado 2018 Nov 23]; 17(4):758-64. Disponivel em: https://www.redalyc.org/ $\mathrm{html} / 714 / 71411240017 /$

8. The Joint Commission Center for Transforming Healthcare: Targeted Solutions Tool $\circledast$ for Preventing Falls; 2015.

9. Hamers JP, Huizing AR. Why do we use physical restraints in the elderly? Z Gerontol Geriatr [Internet]. 2005 [citado 2018 Nov 25]; 38(1):19-25. Disponível em: https://link.springer.com/article/10.1007/s00391-005-0286-x 10. Evans D, Woods J, Lambert L. A review of physical restraint minimization in the acute and residential care settings. J Adv Nurs [Internet]. 2002 [citado 2018 Nov 25]; 40(6):616-25. Disponível em: https://onlinelibrary. wiley.com/doi/full/10.1046/j.1365-2648.2002.02422.x

11. Berzlanovich A, Schöpfer J, Keil W. Deaths due to physical restraint. Dtsch Arztebl Int [Internet]. 2012 [citado 2018 Jan 09]; 109(3):27. Disponível em: https://www.ncbi.nlm.nih.gov/pubmed/22334818file

12. Donius M, Rader J. Use of siderails: rethinking a standard of practice. J Geront Nurs [Internet]. 1994 [citado 2017 Jan 12]; 20(11):23-7. Disponível em: https://www.ncbi.nlm.nih.gov/pubmed/?term=Donius $\% 20 \mathrm{M} \% 5 \mathrm{BAu}-$ thor\%5D\&cauthor=true\&cauthor_uid $=7995900$

13. Healey F, Oliver D, Milne A, Connelly JB. The effect of bedrails on falls and injury: a systematic review of clinical studies. Age Ageing [Internet]. 2008 [citado 2018 Nov 28]; 37(4):368-78. Disponível em: https://academic.oup.com/ageing/article/37/4/368/41175.

14. Burlington DB. Entrapment hazards with hospital bed side rails. FDA Safety Alert. Rockville: US Dep. of Health and Human Services, Public Health Service; 1995. Disponivel em: http://www.fda.gov/cdrh/bedrails.htlm 15. Felsenthal G. Bedrail palsy: the etiology of bilateral footdrop. MD State Med J [Internet]. 1983 [citado 2018 Nov 20]; 32(3):173-4. Disponível em: https://europepmc.org/abstract/med/6855282

16. Miles SH, Irvine P. Deaths caused by physical restraints. The Gerontologist [Internet]. 1992 [citado 2018 Dez 04]; 32(6):762-6. Disponível em: https://academic.oup.com/gerontologist/article-abstract/32/6/762/604896 17. Parker K, Miles SH. Deaths caused by bedrails. J Am Geriat Soc [Internet]. 1997 [citado 2018 Nov 27]; 45(7):797-802. Disponível em: https:// onlinelibrary.wiley.com/doi/full/10.1111/j.1532-5415.1997.tb01504.x

18. Todd JF, Ruhl CE, Gross TP. Injury and death associated with hospital bed side-rails: reports to the US Food and Drug Administration from 1985-1995. Am J Public Health [Internet]. 1997 [citado 2018 Nov 27]; 87(10):1675-7. Disponível em: https://ajph.aphapublications.org/doi/ abs/10.2105/AJPH.87.10.1675

19. Hanger HC, Ball MC, Wood LA. An analisis of falls in the hospital: can we do without bedrails? J Am Geriatr Soc [Internet]. 1999 [citado 2018 Nov 29]; 47(5):529-31. Disponível em: https://onlinelibrary.wiley.com/doi/ full/10.1111/j.1532-5415.1999.tb02565.x

20. Food and Drug Administration - FDA Safety Alert: Entrapment Hazards with Hospital Bed Side Rails. Rockville (USA): Department of Health and Human Services; 1995.

21. Jehan W. Restraint or protection? The use of bed siderails. Nurs Manag [Internet]. 1999 [citado 2018 Nov 27]; 6(2):9-13. Disponível em: https:// search.proquest.com/openview/834073b3e987f776e9e977fdad1f24ea/1?pq-origsite $=$ gscholar $\& \mathrm{cbl}=29902$

22. Gallinagh $R$, Slevin $E$, Mccormack B. Side rails as physical restraints in the care of older people: a management issue. J Nurs Manag [Internet]. 2002 [citado 2018 Nov 27]; 10(5):299-306. Disponível em: https://onlinelibrary.wiley.com/doi/full/10.1046/j.1365-2834.2002.00319.x

23. Sullivan-Marx EM, Strumpf NE. Restraint-free care for acutely ill patients in the Hospital AACN. Adv Crit Care [Internet]. 1996 [citado 2018 Nov 30]; 7(4):572-8. Disponível em: http://acc.aacnjournals.org/content/7/4/572.short

24. Everitt V, Bridel-Nixon J. The use of bed rails: Principles of patient assessment. Nurs Stand [Internet]. 1997 [citado 2019 Jan 30]; 12(6):44-7. Disponivel em: https://europepmc.org/abstract/med/9407871

25. Shanahan D, Evans A. An audit of bedrail use and implications for practice. British J Nurs [Internet]. 2009 [citado 2018 Nov 27]; 18(4):2327. Disponível em: https://www.magonlinelibrary.com/doi/abs/10.12968/ bjon.2009.18.4.39620

26. Grasso J, Van Leeuwen M, Wiles V, Bennett L, West S. Patient falls from bed and the role of bedrails in the acute care setting. Austr J Adv Nurs [Internet]. 2001 [citado 2018 Nov 30]; 19(2):8. Disponível em: https://search. informit.com.au/documentSummary; $d n=406075010434107$; res=IELAPA 27. Capezuti E, Maislin G, Strumpf N, Evans L. Side rail use and falls among nursing home residents. J Am Geriatr Soc [Internet]. 2002 [citado 2018 Nov 30]; 50(1):90-6. Disponível em: https://onlinelibrary.wiley.com/doi/ full/10.1046/j.1532-5415.2002.50013.x

28. Hammond $M$, Levine J. Bedrails: choosing the best alternative. Geriatr Nurs [Internet]. 1999 [citado 2018 Dez 02]; 20(6):297-300. Disponível em: https://www.sciencedirect.com/science/article/pii/S0197457299001093 29. Sousa P, Mendes W. Segurança do paciente: conhecendo os riscos na organização de saúde. Rio de Janeiro: EAD/ENSP Fio Cruz; 2014.

30. Wagner LM, Capezuti E, Brush B, Boltz M, Renz S, Talerico KA. Description of an advanced practice nursing consultative model to reduce restrictive siderail use in nursing homes. Res Nurs Health [Internet]. 2007 [citado 2018 Dez 02]; 30(2):131-40. Disponível em: https://onlinelibrary. wiley.com/doi/abs/10.1002/nur.20185

31. Foltz-Gray D. Caught short by HCFA's restraining orders (surviving the survey). Contemporary Long Term Care. 1998; 21(35).

32. Hospital BSW. Clinical guidance for the assessment and implementation of bed rails in hospitals, long term care facilities, and home care settings. Critical care nursing quarterly [Internet]. 2003 [citado 2018 Dez 02]; 26(3):244. Disponível em: www.fda.gov/cdrh/beds/

33. Brush BL, Capezuti E. Historical analysis of siderail use in american hospitals. J Nurs Scholarship [Internet]. 2001 [citado 2018 Dez 02]; 33(4):381-5. Disponível em: https://sigmapubs.onlinelibrary.wiley.com/ doi/abs/10.1111/j.1547-5069.2001.00381.x

34. Ali G. Ensuring the safe use of cotsides in patient settings. Prof Nurse (London England) [Internet]. 2000 [citado 2018 Dez 13]; 15(4):278-9. Disponivel em: https://europepmc.org/abstract/med/10983146

35. Si M, Neufeld RR, Dunbar J. Removal of bed rails on a short-term nursing home rehabilitation unit. The Gerontol [Internet]. 1999 [citado 2018 Dez 13]; 39(5):611-5. Disponível em: https://academic.oup.com/gerontologist/article/39/5/611/584555

36. Akobeng AK. Principles of evidence based medicine. Arch Dis Child [Internet]. 2005 [citado 2018 Dez 13]; 90(8):837-40. Disponível em: https:// adc.bmj.com/content/90/8/837.short

37. Flemming K. Critical appraisal. 2. Searchable questions. NT Learn Curve [Internet]. 1999 [citado $2019 \mathrm{Abr} 13$ ]; 3(2):6-7. Disponível em: https:// europepmc.org/abstract/med/10474424

38. Bernardo WM, Nobre MR, Jatene FB. Evidence-based clinical practice: part II-Searching evidence databases. Rev Bras Reum [Internet]. 2004 [citado 2019 Abr 13]; 50(1):104-8. Disponivel em: http://www.scielo.br/ scielo.php?pid=S0482-50042004000600003\&script=sci_arttext 